\title{
Short-term reproducibility of intraocular pressure and ocular perfusion pressure measurements in Chinese volunteers and glaucoma patients
}

Yanlin Gao ${ }^{1}$, Bing Wan², Peiyu Li ${ }^{3}$, Yan Zhang ${ }^{1}$ and Xin Tang ${ }^{1 *}$

\begin{abstract}
Background: To evaluate the short-term reproducibility of diurnal intraocular pressure (IOP) and ocular perfusion pressure (OPP) measurements in normal volunteers, untreated normal-tension glaucoma (NTG) and primary open-angle glaucoma (POAG) patients.

Methods: Fifty-four healthy volunteers (control group), 67 NTG patients and 54 POAG patients were recruited. The IOPs of both eyes were measured with a Goldmann applanation tonometer at 3-h intervals over 2 consecutive days. Blood pressure (BP) measurements were collected at the same times. The mean IOP/OPP, peak IOP/OPP, trough IOP/OPP and IOP/OPP fluctuations on each day were also calculated. The intraclass correlation coefficients (ICCs) were used to evaluate the reproducibilities.

Results: In the control group, the ICCs of mean IOP, peak IOP, trough IOP and IOP fluctuation were $0.921,0.889,0.888$, and 0.661 , respectively, and the ICCs of the mean OPP, peak OPP, trough OPP and OPP fluctuations were $0.962,0.918$, 0.953, and 0.680, respectively. In the NTG group, the ICCs of the mean IOP, peak IOP, trough IOP and IOP fluctuation were $0.862,0.741,0.798$, and 0.290 , respectively, and the ICCS of the mean OPP, peak OPP, trough OPP and OPP fluctuations were $0.947,0.828,0.927$, and -0.008 , respectively. In the POAG group, the ICCs of the mean IOP, peak IOP, trough IOP and IOP fluctuation were $0.857,0.666,0.808$, and 0.546 , respectively, and the ICCs of the mean OPP, peak OPP, trough OPP and OPP fluctuation were $0.934,0.842,0.910$, and 0.093, respectively.

Conclusion: The IOP measurements within a single day were not highly reproducible in the short-term. The normal volunteers exhibited better IOP and OPP reproducibilities than the glaucoma patients. The IOP and OPP fluctuations could not be accurately evaluated based on the IOP or OPP measurements within a single day.

Abbreviations: AGIS, Advanced glaucoma intervention study; BP, Blood pressure; CCT, Central corneal thickness; CLS, Contact lens sensor; DBP, Diastolic blood pressure; GAT, Goldmann applanation tonometer; HR, Heart rate; ICCs, Intraclass correlation coefficients; IOP, Intraocular pressure; MD, Mean deviation; NTG, Normal-tension glaucoma; OHT, Ocular hypertension; OPP, Ocular perfusion pressure; POAG, Primary open-angle glaucoma; SBP, Systolic blood pressure; TSNIT, Temporal, superior, nasal, inferior, and temporal; VF, Visual field
\end{abstract}

\footnotetext{
* Correspondence: 34830277@qq.com

${ }^{1}$ Tianjin Eye Hospital, Tianjin Key Laboratory of Ophthalmology and Vision Science, Clinical College of Ophthalmology, Tianjin Medical University, 4, Gansu Road, Heping District, Tianjin 300020, People's Republic of China Full list of author information is available at the end of the article
} 


\section{Background}

Elevated intraocular pressure (IOP) is an identified risk factor for the progression of glaucoma $[1,2]$. Moreover, IOP fluctuations [3] and the long-term mean IOP [4] are significantly correlated with the development of glaucoma. Glaucoma treatment focuses on IOP reduction. It is very important to assess the IOP level before initiating anti-glaucoma treatment.

Patients with normal-tension glaucoma (NTG) exhibit significantly greater reductions in nocturnal blood pressure (BP) than healthy people $[5,6]$, and vascular factors might be associated with the development of glaucoma [6-9]. IOP [10] and BP $[10,11]$ change over time and exhibit inherent circadian rhythms. The ocular perfusion pressure (OPP) is calculated from the IOP and BP. Lower diastolic OPP has been associated with glaucoma in previous population-based studies $[12,13]$. The mean circadian OPP fluctuation is a consistent clinical risk factor for the severity and development of NTG [6-8]. Relative changes in the diurnal mean OPP have also been identified as a risk factor for the diagnosis of primary open-angle glaucoma (POAG) [9].

Twenty-four-hour IOP measurements are widely used in clinical and research practices. We typically measure IOPs at various time points within a single day based on convenience, time and financial cost. IOP values at the same time points are not always stable. The reproducibilities of 24-h IOP patterns are controversial [14-19]. If 24-h IOP patterns are not highly reproducible, IOP measurements over 2 consecutive days or over the long term are necessary to assess the IOP condition.

In this study, normal volunteers, untreated NTG patients and POAG patients underwent IOP and OPP measurements over 2 consecutive days. We analysed the short-term reproducibilities of the IOP and OPP measurements in the 3 groups.

\section{Methods}

\section{Subjects}

The study adhered to the tenets of the Declaration of Helsinki and was approved by the institutional review board and Ethics Committee of Tianjin Eye Hospital. Informed consent was obtained from each subject. The subjects were recruited in the Tianjin Eye Hospital, Tianjin, China. All subjects were free of treatments with anti-glaucoma medications for at least 4 weeks before the IOP measurements. Subjects with systemic hypertension were excluded. And subjects with systemic administration affected BP or IOP were excluded. None of the subjects had any history of ocular surgery or trauma.

None of the normal volunteers had family histories of glaucoma. The IOP values of the normal volunteers ranged from $8 \mathrm{mmHg}$ to $21 \mathrm{mmHg}$. The volunteers underwent complete ophthalmic examinations and exhibited no signs of ophthalmic disease.

The NTG patients were characterized by IOPs $\leq 21$ $\mathrm{mmHg}$ at all time points, glaucomatous visual field (VF) defects, optic disc damage, an open angle of normal appearance, and the absence of secondary causes for glaucomatous optic disc damage.

The POAG patients were characterized by IOPs $>21$ $\mathrm{mmHg}$ at all time points, glaucomatous VF defects, optic disc damage, an open angle of normal appearance, and the absence of secondary causes of glaucomatous optic disc damage.

All subjects underwent a complete ophthalmic examination including central corneal thickness (CCT) measurements (Pentacam, Oculus, Inc., Wetzlar, Germany) and VF tests using a Humphrey Field Analyzer 750i (30-2 program; Carl Zeiss Meditec, Inc., Dublin, California).

\section{IOP measurements}

The IOPs of both eyes was measured over 2 consecutive days. All subjects were in a sitting position, and the IOPs were measured with a Goldmann applanation tonometer (GAT, Carl Zeiss, Inc., Jena, Germany) at 3-h intervals from 0600 to $2400 \mathrm{~h}$. Two experienced doctors were in charge of the IOP measurements. One doctor measured the IOPs on day 1 (6:00-24:00), and the other doctor, masked to the IOP data on day 1 , measured the IOPs on day 2 (6:00-24:00). The nocturnal period was from 21:00 to 06:00 [20]. In the control group, the IOP data from the eye with the better mean deviation (MD) value of visual field was selected. In the glaucoma patients, the IOP data from the eye with the greater visual field defect based on the MD values was selected.

The mean IOP was defined as the average IOP across all measurements over 2 consecutive days (14 time points). The IOPs at each time point were defined as the average IOPs at same time point across all subjects within each group. The mean IOPs on days 1 and day 2 were defined as the average IOPs of all measurements during each day (6:00-24:00, 7 time points). The peak IOP, trough IOP and IOP fluctuation (the peak IOP minus the trough IOP) were also calculated from the IOP measurements during each day. For each subject, the IOPs at each time point were calculated as the average IOPs over the 2 consecutive days. Furthermore, the time points of the peak IOPs, the maximum daytime IOPs and maximum nighttime IOPs were recorded.

\section{OPP calculation}

The systolic BP (SBP), diastolic BP (DBP) and heart rate (HR) were also measured at same time points from 06:00 to 24:00 h. All subjects were in a sitting position and kept calm for at least $5 \mathrm{~min}$. The BP and HR were measured on the upper left arm with an automated 
sphygmomanometer (OMRON HBP-1300, OMRON Healthcare (China) Co., Ltd.). The mean SBP, DBP, and $\mathrm{HR}$ were defined as the averages of the data over 2 consecutive days (14 time points). The OPPs at each time point were calculated as follows: $\mathrm{OPP}=2 / 3 \times$ $[\mathrm{DBP}+\{1 / 3 \times(\mathrm{SBP}-\mathrm{DBP})\}]-\mathrm{IOP}$ [21]. The main parameters of the OPP were calculated with the same methods used for the IOP.

\section{Statistical analysis}

The gender, eye (right/left), and MD values of the 3 groups were analysed using Kruskal-Wallis $\mathrm{H}$ test. The age, CCT, IOP at diagnosis, mean IOP, mean OPP, mean SBP, mean DBP and mean HR were analysed using one-way analyses of variance (ANOVA). The reproducibilities over the 2 consecutive days were elevated with the intraclass correlation coefficients (ICCs) and Bland-Altman Plots. The ICC indicates the proportion of variance in a measurement that is due to differences among subjects. The $\alpha$ level (type I error) was set at 0.05 . An ICC $\geq 0.75$ was considered indicative of excellent reproducibility, an ICC of $0.4 \leq$ ICC $<0.75$ was considered indicative of fair reproducibility, and an ICC of ICC $<0.4$ was considered indicative of poor reproducibility [22]. Negative ICC values indicate greater within-subject variability than between-subject variability and represent an agreement that is below that expected by chance alone [17]. The statistical analyses were performed with SPSS (version 16.0; SPSS Inc., Chicago, IL). BlandAltman Plots were created with Medcalc (Version 11.4.2.0; Medcalc Software Inc., Mariakerke, Belgium). $P<0.05$ was considered statistically significant.

\section{Results}

\section{Subject characteristics}

Fifty-four normal volunteers (control group), 67 NTG patients and 54 POAG patients were recruited, and the characteristics of each group are shown in Table 1 . The POAG group exhibited a significantly higher mean IOP over the 2 consecutive days $(28.3 \pm 2.1 \mathrm{mmHg})$ than the control $(14.3 \pm 1.9 \mathrm{mmHg})$ and NTG groups (13.9 \pm $1.6 \mathrm{mmHg}, P<0.001)$. The mean IOPs over 2 consecutive days exhibited no significant difference between the control and NTG groups $(P=0.271)$. The POAG group exhibited a significantly lower mean OPP over the 2 consecutive days $(29.6 \pm 4.5 \mathrm{mmHg})$ than the control $(44.7 \pm 4.8 \mathrm{mmHg})$ and NTG groups $(43.5 \pm 5.2 \mathrm{mmHg}$, $P<0.001)$. The mean OPP over 2 consecutive days exhibited no significant difference between the control and NTG groups $(P=0.186)$. The ages, eyes (right/left), genders, CCTs, mean SBPs, mean DBPs and mean HRs over the 2 consecutive days exhibited no significant differences within the 3 groups.

\section{ICC estimates of the IOPs at each time point}

The ICC estimates of the IOPs at each time point across the 2 days are presented in Table 2. In the control group, the ICCs ranged from 0.688 (15:00) to $0.876(6: 00)$. The ICC values exhibited fair to excellent reproducibility. In the NTG group, the ICCs ranged from 0.347 (15:00) to 0.762 (21:00) and indicated large variations in reproducibility from poor to excellent at different time points. In the POAG group, the ICCs ranged from 0.595 (21:00) to 0.787 (9:00 and 12:00), and these ICC values indicated fair to excellent reproducibility.

\section{ICC estimates of the OPPs at each time point}

The ICC estimates of the OPPs at each time point across the 2 days are presented in Table 3. In the control group, the ICCs ranged from 0.784 (24:00) to 0.896 (6:00) and indicated excellent reproducibility. In the NTG group, the ICCs ranged from 0.720 (18:00) to 0.891 (21:00). Most of these ICC values indicated excellent reproducibility, and only one value $(0.720,18: 00)$ indicated fair reproducibility. In the POAG group, the ICCs ranged from 0.714 (18:00) to 0.878 (9:00), and most of these ICC values indicated excellent reproducibility.

\section{ICC estimates for the main parameters of diurnal curves}

The diurnal IOP and diurnal OPP curves were plotted according to the IOP and OPP data at each time point (Fig. 1). The mean IOP/OPP, peak IOP/OPP, trough IOP/OPP and IOP/OPP fluctuation values were calculated from the diurnal curves. The ICC estimates of these parameters for the 2 days are presented in Table 4.

In the control group, the IOP reproducibility was greatest for the mean IOP $(\mathrm{ICC}=0.921)$ followed by the peak IOP $(\mathrm{ICC}=0.889)$ and the trough IOP $(\mathrm{ICC}=0.888)$. The IOP fluctuation exhibited the lowest reproducibility $(\mathrm{ICC}=0.661)$. The reproducibility of the OPP was highest for the mean OPP (ICC = 0.962) followed by the trough OPP $(\mathrm{ICC}=0.953)$ and the peak OPP $(\mathrm{ICC}=0.918)$. The OPP fluctuation exhibited the lowest reproducibility $(\mathrm{ICC}=0.680)$.

In the NTG group, the IOP reproducibility was highest for the mean IOP (ICC $=0.862$ ) followed by the trough IOP $(\mathrm{ICC}=0.798)$ and the peak IOP (ICC $=0.741)$. The IOP fluctuation exhibited the lowest reproducibility $(\mathrm{ICC}=0.290)$. The reproducibility of the OPP was highest for the mean OPP (ICC = 0.947) followed by the trough OPP $(\mathrm{ICC}=0.927)$ and the peak OPP ( $\mathrm{ICC}=0.828$ ). The OPP fluctuation exhibited the lowest reproducibility $(\mathrm{ICC}=-0.008)$.

In the POAG group, the IOP reproducibility was highest for the mean IOP (ICC $=0.857$ ) followed by the trough IOP ( $\mathrm{ICC}=0.808)$ and the peak IOP $(\mathrm{ICC}=0.666)$. The IOP fluctuation exhibited the lowest reproducibility $(\mathrm{ICC}=0.546)$. The reproducibility of the OPP was 
Table 1 Characteristics of Subjects

\begin{tabular}{|c|c|c|c|c|}
\hline Characteristic & Control & NTG & POAG & $P$ value \\
\hline Number & 54 & 67 & 54 & \\
\hline Gender (Male/Female) $^{a}$ & $31 / 23$ & $37 / 30$ & $32 / 22$ & 0.965 \\
\hline Eye (Right/left) ${ }^{\mathrm{a}}$ & $32 / 22$ & $34 / 33$ & $26 / 28$ & 0.479 \\
\hline Age(yrs) & $50.7 \pm 14.0$ & $52.2 \pm 13.6$ & $51.4 \pm 11.7$ & 0.830 \\
\hline IOP at diagnosis (mmHg) & $15.5 \pm 2.5$ & $15.2 \pm 2.1$ & $28.6 \pm 3.1$ & $<0.001$ \\
\hline Mean deviation $(\mathrm{dB})^{\mathrm{a}}$ & $-0.51 \pm 1.0$ & $-9.32 \pm 4.91$ & $-9.60 \pm 5.52$ & $<0.001$ \\
\hline $\mathrm{CCT}(\mu \mathrm{m})$ & $549.2 \pm 30.5$ & $542.6 \pm 31.3$ & $555.0 \pm 26.7$ & 0.064 \\
\hline Mean IOP (mmHg) & $14.3 \pm 1.9$ & $13.9 \pm 1.6$ & $28.3 \pm 2.1$ & $<0.001$ \\
\hline Mean OPP $(\mathrm{mmHg})$ & $44.7 \pm 4.8$ & $43.5 \pm 5.2$ & $29.6 \pm 4.5$ & $<0.001$ \\
\hline Mean SBP (mmHg) & $118.7 \pm 9.4$ & $116.3 \pm 10.2$ & $117.4 \pm 9.2$ & 0.395 \\
\hline Mean DBP $(\mathrm{mmHg})$ & $73.4 \pm 6.8$ & $70.9 \pm 7.6$ & $71.7 \pm 6.0$ & 0.139 \\
\hline Mean HR & $68.8 \pm 8.1$ & $68.6 \pm 7.3$ & $68.4 \pm 7.1$ & 0.969 \\
\hline
\end{tabular}

NTG normal tension glaucoma, $P O A G$ primary open-angle glaucoma, IOP intraocular pressure; CCT central corneal thickness, OPP ocular perfusion pressure; SBP systolic blood pressure, $D B P$ diastolic blood pressure, $H R$ heart rate

Data are expressed as mean \pm standard deviation

${ }^{a}$ Kruskal-Wallis test; other parameters were analyzed using one-way analysis of variance

highest for the mean OPP (ICC = 0.934) followed by the trough OPP $(\mathrm{ICC}=0.910)$ and the peak OPP $(\mathrm{ICC}=0.842)$. The OPP fluctuation exhibited the lowest reproducibility $(\mathrm{ICC}=0.093)$.

For each group, the mean IOPs and mean OPPs exhibited the greatest reproducibilities, whereas the IOP and OPP fluctuations elicited the poorest reproducibilities.

\section{Bland-Altman plots of main parameters of the diurnal curves}

Figure 2 to 7 present Bland-Altman plots comparing the main parameters of the individuals over 2 days in the control, NTG and POAG groups.

For the control group, the mean differences between 2 days were $0 \mathrm{mmHg}$ for the mean IOP and trough IOP, $0.4 \mathrm{mmHg}$ for the peak IOP, $0.5 \mathrm{mmHg}$ for the IOP fluctuation and peak OPP, $0.7 \mathrm{mmHg}$ for the mean OPP, $0.8 \mathrm{mmHg}$ for the trough OPP and $-0.3 \mathrm{mmHg}$ for the OPP fluctuation (Figs. 2 and 3).
For the NTG group, the mean differences between 2 days were $0.1 \mathrm{mmHg}$ for the trough IOP and IOP fluctuation, $0.2 \mathrm{mmHg}$ for the mean IOP, peak IOP, mean OPP and trough OPP, $0.3 \mathrm{mmHg}$ for the OPP fluctuation and $0.5 \mathrm{mmHg}$ for the peak OPP (Figs. 4 and 5).

For the POAG group, the mean differences between the 2 days were $0.1 \mathrm{mmHg}$ for the peak IOP and peak OPP, $0.2 \mathrm{mmHg}$ for the OPP fluctuation, $0.3 \mathrm{mmHg}$ for the mean IOP, $0.5 \mathrm{mmHg}$ for the trough IOP and trough OPP, $0.9 \mathrm{mmHg}$ for the mean OPP and $-0.4 \mathrm{mmHg}$ for the IOP fluctuation (Figs. 6 and 7).

\section{Test-retest differences in the main parameters of the diurnal curves}

The test-retest differences in the main parameters in the different intervals were calculated and are presented in Table 5 and 6.

The test-retest difference in the mean IOPs of the control, NTG, and POAG groups fell within $\pm 2 \mathrm{mmHg}$ in

Table 2 Intraclass Correlation Coefficient Estimates of Intraocular Pressure at Each Time Point

\begin{tabular}{|c|c|c|c|c|c|c|c|c|c|}
\hline \multirow{2}{*}{$\begin{array}{l}\text { Time } \\
\text { point }\end{array}$} & \multirow[b]{2}{*}{ Day 1} & \multicolumn{3}{|l|}{ Control } & \multicolumn{3}{|l|}{ NTG } & \multicolumn{2}{|l|}{ POAG } \\
\hline & & Day 2 & $I C C^{a}$ & Day 1 & Day 2 & $I C C^{a}$ & Day 1 & Day 2 & $\overline{I C C^{a}}$ \\
\hline 6:00 & $15.5 \pm 2.6$ & $14.8 \pm 2.7$ & 0.876 & $14.6 \pm 2.3$ & $13.9 \pm 2.3$ & 0.696 & $29.1 \pm 3.1$ & $28.6 \pm 2.9$ & 0.744 \\
\hline 9:00 & $14.2 \pm 2.5$ & $14.6 \pm 2.5$ & 0.815 & $13.7 \pm 2.0$ & $13.8 \pm 2.4$ & 0.646 & $28.5 \pm 3.1$ & $28.1 \pm 3.0$ & 0.787 \\
\hline 12:00 & $14.9 \pm 2.6$ & $15.1 \pm 2.6$ & 0.802 & $14.4 \pm 2.1$ & $14.6 \pm 2.1$ & 0.694 & $29.2 \pm 3.0$ & $29.1 \pm 3.0$ & 0.787 \\
\hline 15:00 & $14.0 \pm 2.1$ & $14.0 \pm 2.5$ & 0.688 & $14.0 \pm 2.0$ & $13.8 \pm 2.1$ & 0.347 & $28.3 \pm 3.1$ & $27.9 \pm 2.6$ & 0.752 \\
\hline 18:00 & $14.7 \pm 2.2$ & $14.8 \pm 2.2$ & 0.736 & $14.6 \pm 2.2$ & $14.4 \pm 2.0$ & 0.710 & $29.2 \pm 2.9$ & $28.7 \pm 2.4$ & 0.708 \\
\hline 21:00 & $13.1 \pm 2.4$ & $13.2 \pm 2.3$ & 0.756 & $13.5 \pm 2.5$ & $13.2 \pm 2.2$ & 0.762 & $27.6 \pm 3.3$ & $27.3 \pm 2.4$ & 0.595 \\
\hline 24:00 & $13.3 \pm 2.6$ & $13.3 \pm 2.2$ & 0.714 & $13.0 \pm 2.3$ & $13.0 \pm 2.4$ & 0.523 & $27.6 \pm 2.7$ & $27.2 \pm 3.2$ & 0.656 \\
\hline
\end{tabular}

Data are expressed as mean \pm standard deviation

NTG normal tension glaucoma, POAG primary open-angle glaucoma, ICC intraclass correlation coefficient

${ }^{a}$ All ICC measurements, $P<0.001$ 
Table 3 Intraclass Correlation Coefficient Estimates of Ocular Perfusion Pressure at Each Time Point

\begin{tabular}{|c|c|c|c|c|c|c|c|c|c|}
\hline \multirow{2}{*}{$\begin{array}{l}\text { Time } \\
\text { point }\end{array}$} & \multirow[b]{2}{*}{ Day 1} & \multicolumn{3}{|l|}{ Control } & \multicolumn{3}{|l|}{ NTG } & \multicolumn{2}{|l|}{ POAG } \\
\hline & & Day 2 & $I C C^{a}$ & Day 1 & Day 2 & $I C C^{a}$ & Day 1 & Day 2 & $\mathrm{ICC}^{\mathrm{a}}$ \\
\hline 6:00 & $45.6 \pm 6.5$ & $42.8 \pm 6.7$ & 0.896 & $42.9 \pm 6.8$ & $42.3 \pm 6.1$ & 0.841 & $30.7 \pm 6.0$ & $28.7 \pm 5.1$ & 0.857 \\
\hline 9:00 & $44.9 \pm 6.0$ & $43.5 \pm 5.4$ & 0.787 & $44.0 \pm 5.8$ & $42.7 \pm 6.2$ & 0.880 & $29.6 \pm 5.0$ & $28.1 \pm 5.3$ & 0.878 \\
\hline 12:00 & $43.4 \pm 6.7$ & $43.4 \pm 6.8$ & 0.889 & $41.9 \pm 5.4$ & $43.0 \pm 5.6$ & 0.797 & $28.6 \pm 5.8$ & $28.4 \pm 5.5$ & 0.734 \\
\hline 15:00 & $44.6 \pm 6.2$ & $43.7 \pm 6.1$ & 0.791 & $42.7 \pm 6.8$ & $42.6 \pm 6.4$ & 0.832 & $29.1 \pm 5.7$ & $29.3 \pm 5.8$ & 0.805 \\
\hline 18:00 & $46.1 \pm 6.6$ & $45.5 \pm 6.3$ & 0.882 & $45.2 \pm 6.5$ & $43.9 \pm 6.7$ & 0.720 & $30.3 \pm 6.2$ & $29.2 \pm 6.1$ & 0.714 \\
\hline 21:00 & $45.8 \pm 5.8$ & $46.0 \pm 5.7$ & 0.867 & $43.7 \pm 7.0$ & $44.3 \pm 5.2$ & 0.891 & $30.9 \pm 6.0$ & $30.1 \pm 5.4$ & 0.818 \\
\hline 24:00 & $45.5 \pm 5.7$ & $45.9 \pm 5.5$ & 0.784 & $44.5 \pm 6.5$ & $45.1 \pm 5.6$ & 0.832 & $31.3 \pm 5.3$ & $30.3 \pm 5.3$ & 0.757 \\
\hline
\end{tabular}

Data are expressed as mean \pm standard deviation

NTG normal tension glaucoma, POAG primary open-angle glaucoma, ICC intraclass correlation coefficient

${ }^{a}$ All ICC measurements, $P<0.001$

92.6, 91.0 and $81.5 \%$ of the subjects, respectively. The corresponding test-retest difference in the peak IOPs of the 3 group fell within $\pm 2 \mathrm{mmHg}$ in $92.6,88.1$ and $77.8 \%$ of the subjects. The corresponding test-retest differences in the trough IOPs fell within $\pm 2 \mathrm{mmHg}$ in 94.4, 85.1 and $74.1 \%$ of the subjects. The test-retest differences in the IOP fluctuations in the control, NTG, and POAG groups fell within $\pm 2 \mathrm{mmHg}$ in $88.9,80.6$ and $75.9 \%$ of the subjects, respectively.

The test-retest differences in the mean OPPs of the control, NTG, and POAG groups fell within $\pm 5 \mathrm{mmHg}$ in 98.1, 95.5 and $100 \%$ of the subjects, respectively. The corresponding test-retest differences in the peak OPPs fell within $\pm 5 \mathrm{mmHg}$ in $88.9,82.1$ and $75.9 \%$ of the subjects. The corresponding test-retest differences in the trough OPPs fell within $\pm 5 \mathrm{mmHg}$ in $92.6,94.0$ and $92.6 \%$ of the subjects. The test-retest differences in the IOP fluctuations in the control, NTG, and POAG groups fell within $\pm 5 \mathrm{mmHg}$ in 92.6, 71.6 and $75.9 \%$ of the subjects, respectively.

\section{Daytime vs. nighttime IOPs/OPPs}

The distributions of the peak IOP/OPP times are illustrated in Table 7. The peak IOP time points with the greatest frequencies were 6:00 for the control group (46.3\%), 18:00 for the NTG group (34.3\%) and 12:00 for the POAG group (29.6\%). The times with the greatest frequencies of peak OPPs were 18:00 in the control group (20.4\%), 24:00 in the NTG group $(28.4 \%)$ and $24: 00$ in the POAG group (27.8 \%).

Table 8 illustrates how well the maximum daytime pressures predicted the nighttime measurements at various pressure levels. The table indicates that the nighttime pressures of $76.0 \%$ of the normal volunteers, $71.7 \%$ of the NTG patients and $59.3 \%$ of the POAG patients were within $1.0 \mathrm{mmHg}$ of the maximum daytime IOP readings. Furthermore, the nighttime pressures of $77.8 \%$ of the normal volunteers, $62.7 \%$ of the NTG patients and $66.6 \%$ of the POAG patients were within $2.0 \mathrm{mmHg}$ of the maximum daytime OPP readings.

The POAG patients had significantly greater nighttime OPPs $(30.3 \pm 5.1 \mathrm{mmHg})$ than daytime OPPs $(29.1 \pm 5.1 \mathrm{mmHg}, P=0.020)$, whereas the control and NTG group exhibited no differences between the daytime and nighttime OPPs.

\section{Discussion}

In this study, we collected IOP measurements over 2 consecutive days that revealed the following findings: (1)

\section{a}

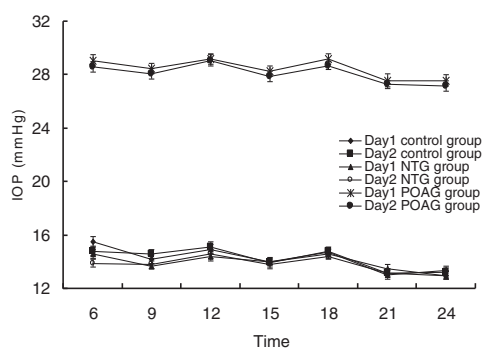

b

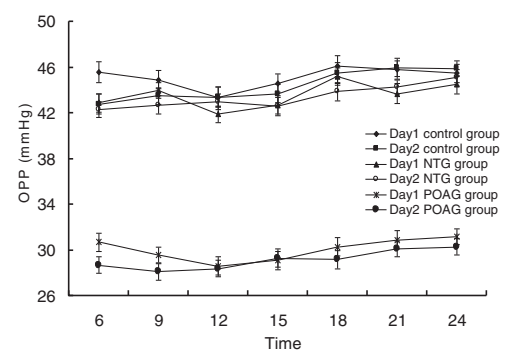

Fig. 1 Diurnal Curves of the 3 groups. The diurnal IOP (a) and diurnal OPP curves (b) were plotted according to the IOP and OPP data at each time point. IOPs and OPPs changed with time and showed a typical circadian rhythm. The peak IOPs did not occur at same time points over the 2 days in the normal volunteers or glaucoma patients. The peak OPPs occurred at same time points (24:00) only in the POAG patients 
Table 4 Intraclass Correlation Coefficient Estimates for the Main Parameters in diurnal curves

\begin{tabular}{|c|c|c|c|c|c|c|c|c|c|}
\hline \multirow[t]{2}{*}{ Parameters } & \multirow[b]{2}{*}{ Day 1} & \multicolumn{3}{|l|}{ Control } & \multicolumn{3}{|l|}{ NTG } & \multicolumn{2}{|l|}{ POAG } \\
\hline & & Day 2 & $I C C^{a}$ & Day 1 & Day 2 & $I C C^{a}$ & Day 1 & Day 2 & $\mathrm{ICC}^{\mathrm{a}}$ \\
\hline Mean IOP & $14.3 \pm 2.0$ & $14.3 \pm 2.0$ & 0.921 & $14.0 \pm 1.7$ & $13.8 \pm 1.7$ & 0.862 & $28.5 \pm 2.4$ & $28.1 \pm 2.0$ & 0.857 \\
\hline Peak IOP & $16.8 \pm 2.2$ & $16.4 \pm 2.2$ & 0.889 & $16.3 \pm 1.9$ & $16.0 \pm 1.7$ & 0.741 & $31.2 \pm 2.4$ & $31.1 \pm 2.1$ & 0.666 \\
\hline Trough IOP & $12.0 \pm 2.0$ & $12.0 \pm 2.0$ & 0.888 & $11.8 \pm 1.7$ & $11.7 \pm 2.1$ & 0.798 & $25.8 \pm 2.6$ & $25.3 \pm 2.3$ & 0.808 \\
\hline IOP Fluctuation & $4.8 \pm 1.4$ & $4.3 \pm 1.4$ & 0.661 & $4.5 \pm 1.4$ & $4.4 \pm 1.5$ & 0.290 & $5.4 \pm 1.8$ & $5.7 \pm 2.0$ & 0.546 \\
\hline Mean OPP & $45.1 \pm 5.3$ & $44.4 \pm 5.2$ & 0.962 & $43.6 \pm 5.5$ & $43.4 \pm 5.1$ & 0.947 & $30.1 \pm 4.7$ & $29.2 \pm 4.5$ & 0.934 \\
\hline Peak OPP & $49.8 \pm 5.5$ & $49.3 \pm 5.7$ & 0.918 & $48.7 \pm 6.2$ & $48.2 \pm 5.4$ & 0.828 & $34.8 \pm 5.3$ & $34.1 \pm 4.6$ & 0.842 \\
\hline Trough OPP & $40.1 \pm 5.9$ & $39.3 \pm 5.6$ & 0.953 & $38.7 \pm 5.5$ & $38.5 \pm 5.4$ & 0.927 & $25.2 \pm 4.8$ & $24.7 \pm 4.7$ & 0.910 \\
\hline OPP Fluctuation & $9.7 \pm 3.4$ & $10.0 \pm 3.0$ & 0.680 & $10.0 \pm 4.4$ & $9.7 \pm 3.4$ & -0.008 & $9.7 \pm 3.3$ & $9.5 \pm 2.8$ & 0.093 \\
\hline
\end{tabular}

Data are expressed as mean \pm standard deviation

$I O P$ intraocular pressure, OPP ocular perfusion pressure, ICC intraclass correlation coefficient

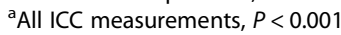

POAG group exhibited larger IOP fluctuations than the control and NTG groups; (2) the POAG group exhibited significantly lower OPPs than the control and NTG groups; (3) the control group exhibited better IOP reproducibility across the time points than the glaucoma groups; (4) the OPPs across the time points in each group exhibited a tendency towards excellent reproducibility; (5) the mean and trough IOPs of each group exhibited excellent reproducibilities, the peak IOP of the control group exhibited better excellent reproducibility than those of the glaucoma groups, and the IOP fluctuations exhibited fair or poor reproducibilities; (6) the mean OPP, peak OPP and trough OPP exhibited excellent reproducibilities, while the OPP fluctuations exhibited fair or poor reproducibilities; and (7) the nighttime readings of the majority of the subjects were within $1.0 \mathrm{mmHg}$ of the maximum daytime IOP reading and $2.0 \mathrm{mmHg}$ of the maximum daytime OPP.

Typical patterns of diurnal IOP and OPP fluctuations were observed in the present study [21]. The peak IOPs did not occur at same time points over the 2 days in the normal volunteers or glaucoma patients. The peak OPPs occurred at same time points (24:00) only in the POAG patients. The greatest frequencies of peak IOPs occurred

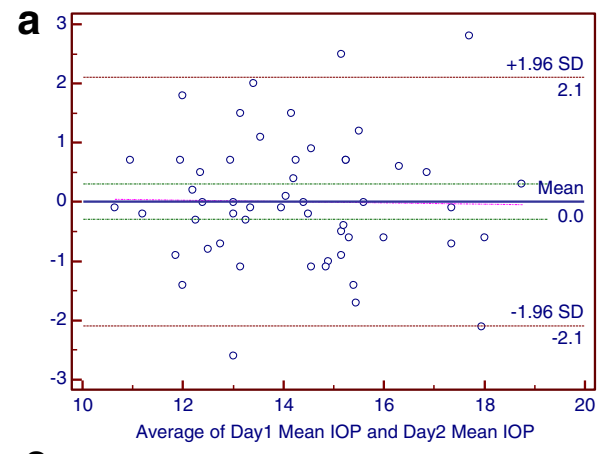

C

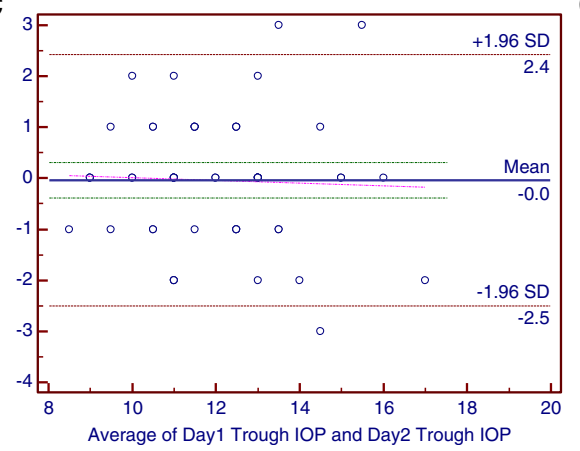

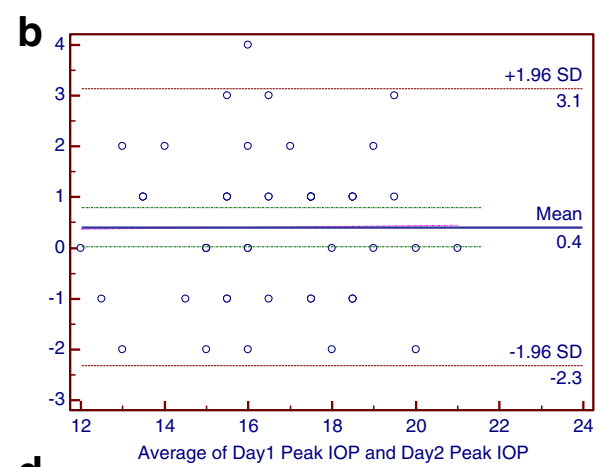

d

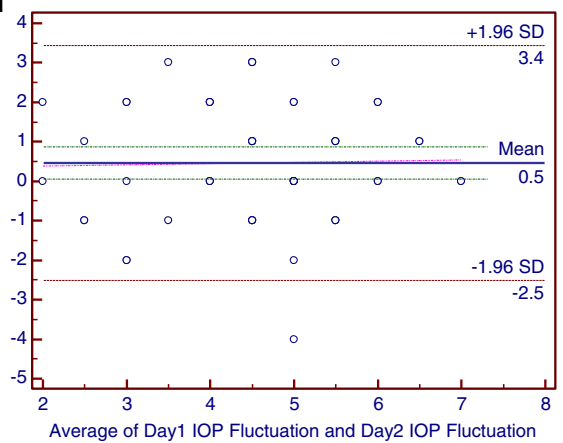

Fig. 2 The Bland-Altman Plots for Intraocular Pressure (IOP) in the Control Group. Part $\mathbf{a}, \mathbf{b}, \mathbf{c}$ and $\mathbf{d}$ respectively reflected the individual test-retest difference conditions of mean IOP, peak IOP, trough IOP and IOP fluctuation 

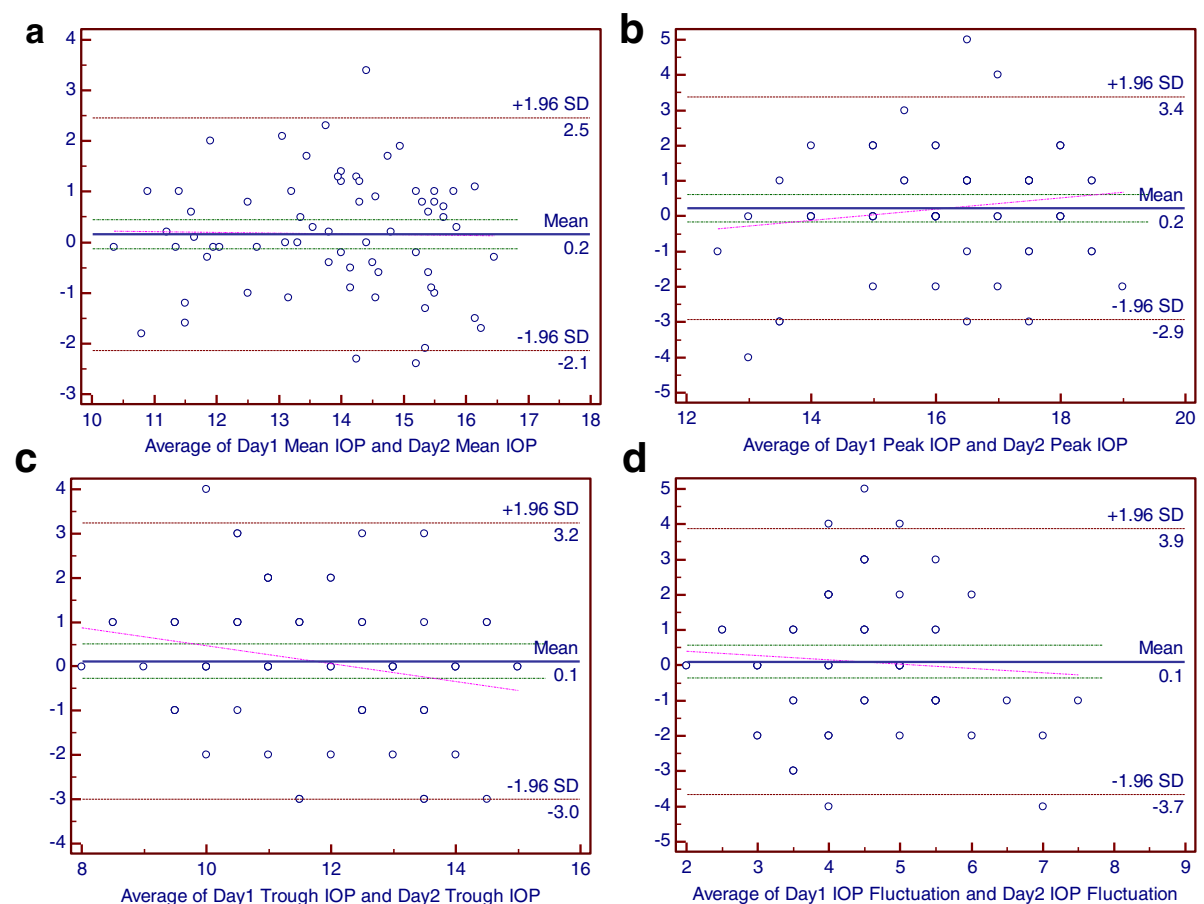

d

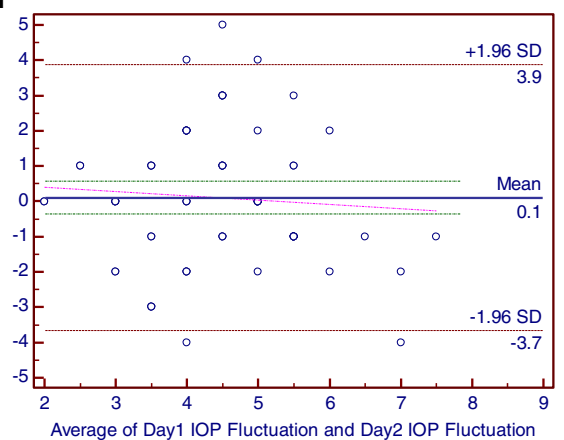

Fig. 3 The Bland-Altman Plots for Intraocular Pressure (IOP) in the Normal-Tension Glaucoma Group. Part a, b, c and $\mathbf{d}$ respectively reflected the individual test-retest difference conditions of mean IOP, peak IOP, trough IOP and IOP fluctuation
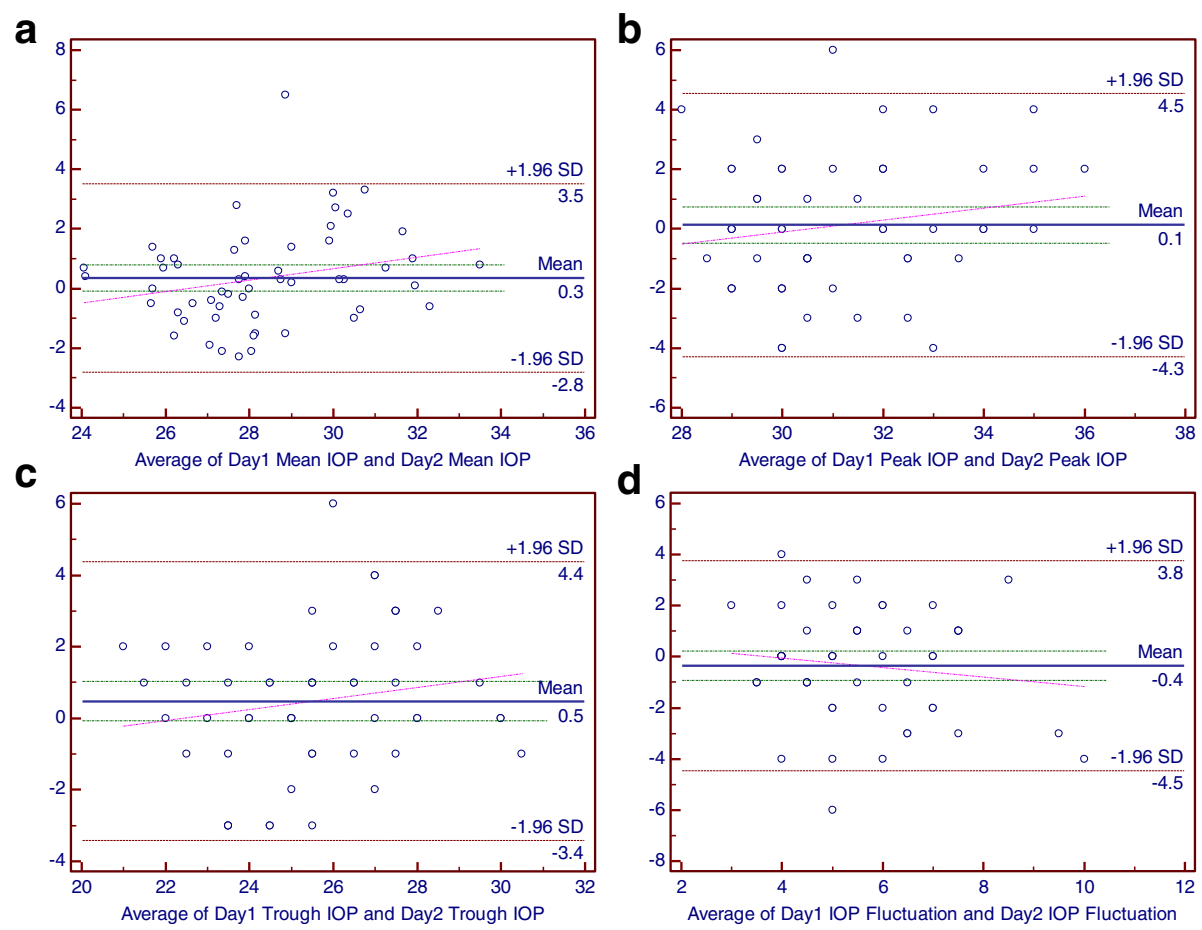

Fig. 4 The Bland-Altman Plots for Intraocular Pressure (IOP) in the Primary Open-Angle Glaucoma Group. Part a, b, c and $\mathbf{d}$ respectively reflected the individual test-retest difference conditions of mean IOP, peak IOP, trough IOP and IOP fluctuation 

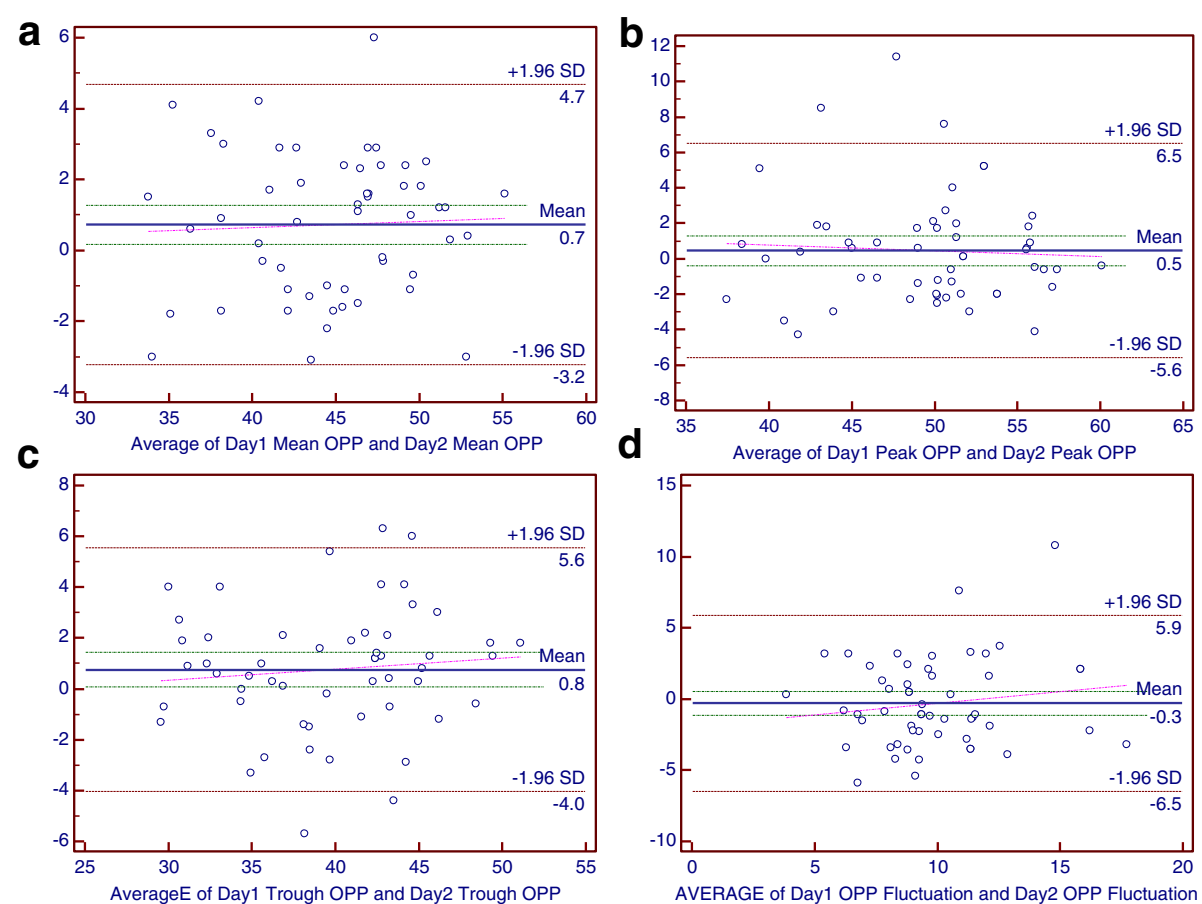

Fig. 5 The Bland-Altman Plots for Ocular Perfusion Pressure (OPP) in the Control Group. Part $\mathbf{a}, \mathbf{b}, \mathbf{c}$ and $\mathbf{d}$ respectively reflected the individual test-retest difference conditions of mean OPP, peak OPP, trough OPP and OPP fluctuation
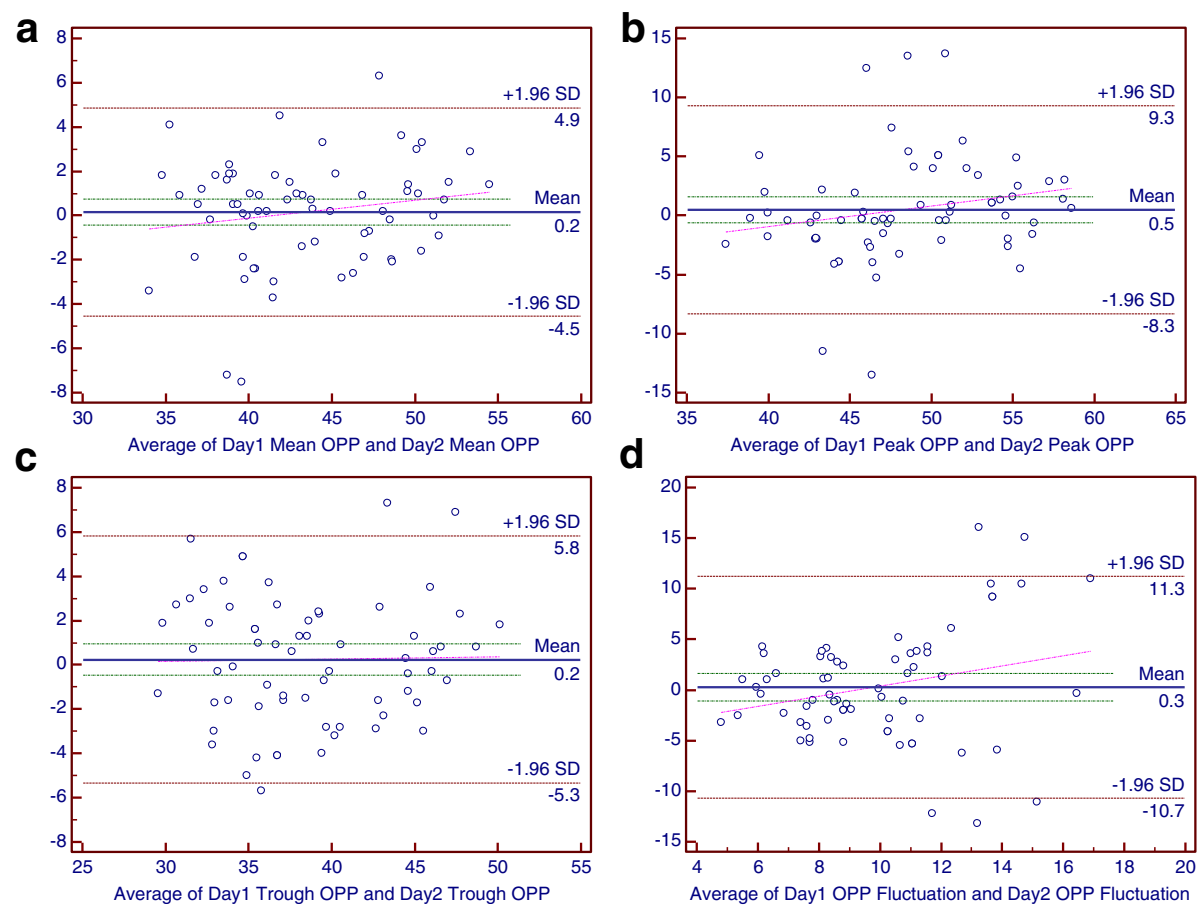

d

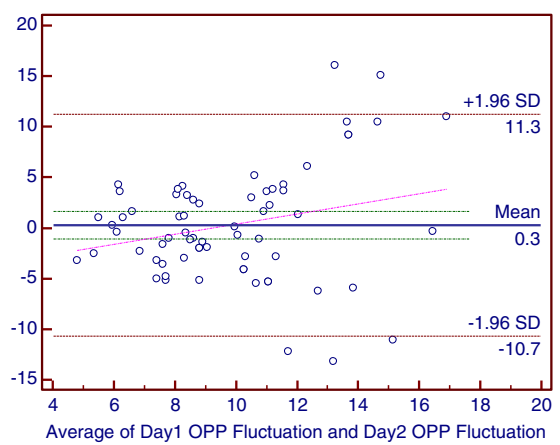

Fig. 6 The Bland-Altman Plots for Ocular Perfusion Pressure (OPP) in the Normal-Tension Glaucoma Group. Part a, b, c and $\mathbf{d}$ respectively reflected the individual test-retest difference conditions of mean OPP, peak OPP, trough OPP and OPP fluctuation 

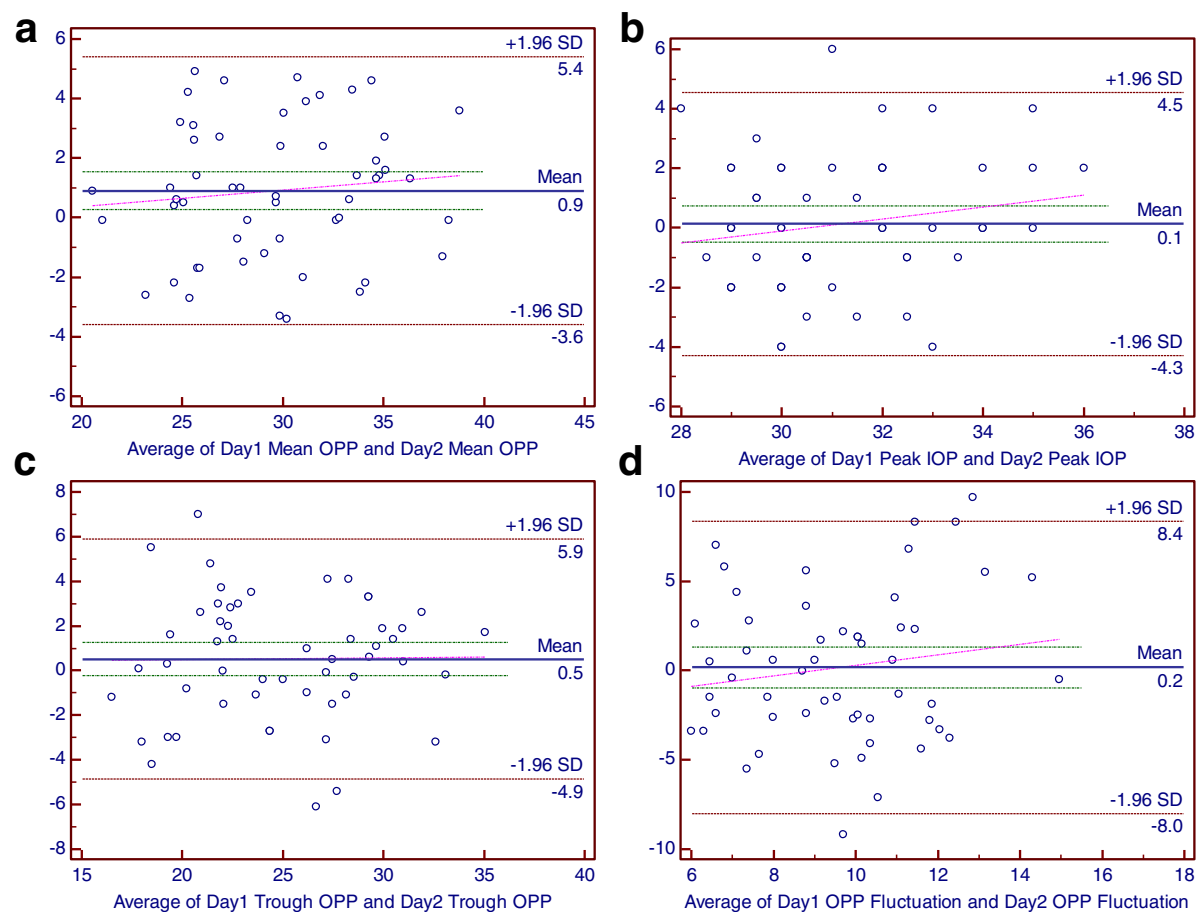

Fig. 7 The Bland-Altman Plots for Ocular Perfusion Pressure (OPP) in the Primary Open-Angle Glaucoma Group. Part $\mathbf{a}$, b, $\mathbf{c}$ and $\mathbf{d}$ respectively reflected the individual test-retest difference conditions of mean OPP, peak OPP, trough OPP and OPP fluctuation

at different time points, and the greatest highest frequencies of peak OPPs occurred at same time point (24:00) in the glaucoma patients. Quaranta et al. reported that the great majority of untreated glaucoma patients exhibit their peak IOP during the daytime, and the highest nighttime IOP values of approximately $70 \%$ of patients are within $1.0 \mathrm{mmHg}$ of the highest daytime IOP values [20]. In this study, some subjects exhibited peak IOPs in the morning (06:00), and the highest nighttime IOPs of $59.3 \%$ of the POAG patients were within $1 \mathrm{mmHg}$ of the highest daytime IOPs. The peak IOPs most frequently occurred outside of office hours. Furthermore, the characteristics of the 24-h IOP curves were not correctly predicted based on the IOP data collected during office hours [23]. The peak IOPs were not highly reproducible, and the peak IOPs times were quite different, and these differences may be attributable to differences in the body positions, tonometers and circadian CCT fluctuations.

The short- and long-term reproducibilities of IOP measurements have been evaluated in some studies. Realini et al. measured the IOPs of healthy individuals with a GAT at $2 \mathrm{~h}$ intervals from 8:00 to 20:00 during 2 visits spaced 1 week apart. The eyes of the healthy individuals did not exhibit a sustained and reproducible diurnal IOP pattern across the 2 visits [14]. Later, Realini et al. measured the IOPs of treated POAG patients at

Table 5 Proportions of Parameter Test-Retest Differences within Different Intervals between Two Days

\begin{tabular}{|c|c|c|c|c|c|c|c|c|c|c|c|c|}
\hline \multirow[t]{2}{*}{ Interval } & \multirow[b]{2}{*}{$\begin{array}{l}\text { Mean } \\
\text { IOP }\end{array}$} & \multicolumn{4}{|c|}{ Control } & \multicolumn{4}{|l|}{ NTG } & \multicolumn{3}{|l|}{ POAG } \\
\hline & & $\begin{array}{l}\text { Peak } \\
\text { IOP }\end{array}$ & $\begin{array}{l}\text { Trough } \\
\text { IOP }\end{array}$ & $\begin{array}{l}\text { IOP } \\
\text { Fluctuation }\end{array}$ & $\begin{array}{l}\text { Mean } \\
\text { IOP }\end{array}$ & $\begin{array}{l}\text { Peak } \\
\text { IOP }\end{array}$ & $\begin{array}{l}\text { Trough } \\
\text { IOP }\end{array}$ & $\begin{array}{l}\text { IOP } \\
\text { Fluctuation }\end{array}$ & $\begin{array}{l}\text { Mean } \\
\text { IOP }\end{array}$ & $\begin{array}{l}\text { Peak } \\
\text { IOP }\end{array}$ & $\begin{array}{l}\text { Trough } \\
\text { IOP }\end{array}$ & $\begin{array}{l}\text { IOP } \\
\text { Fluctuation }\end{array}$ \\
\hline$\leq 1 \mathrm{mmHg}$ & 70.4 & 74.1 & 79.6 & 64.8 & 62.7 & 70.1 & 68.7 & 58.2 & 59.3 & 44.4 & 57.4 & 55.6 \\
\hline$\leq 2 \mathrm{mmHg}$ & 92.6 & 92.6 & 94.4 & 88.9 & 91.0 & 88.1 & 85.1 & 80.6 & 81.5 & 77.8 & 74.1 & 75.9 \\
\hline$\leq 3 \mathrm{mmHg}$ & 100 & 98.1 & 98.1 & 98.1 & 98.5 & 95.5 & 98.5 & 92.5 & 94.4 & 85.2 & 94.4 & 88.9 \\
\hline$\leq 4 \mathrm{mmHg}$ & & 100 & 100 & 100 & 100 & 98.5 & 100 & 98.5 & 98.1 & 98.1 & 98.1 & 98.1 \\
\hline$\leq 5 \mathrm{mmHg}$ & & & & & & 100 & & 100 & 98.1 & 98.1 & 98.1 & 98.1 \\
\hline$\leq 6 \mathrm{mmHg}$ & & & & & & & & & 98.1 & 100 & 100 & 100 \\
\hline$\leq 7 \mathrm{mmHg}$ & & & & & & & & & 100 & & & \\
\hline
\end{tabular}


Table 6 Proportions of Parameter Test-Retest Differences within Different Intervals between Two Days

\begin{tabular}{|c|c|c|c|c|c|c|c|c|c|c|c|c|}
\hline \multirow[t]{2}{*}{ Interval } & \multirow[b]{2}{*}{$\begin{array}{l}\text { Mean } \\
\text { OPP }\end{array}$} & \multicolumn{4}{|c|}{ Control } & \multicolumn{4}{|l|}{ NTG } & \multicolumn{3}{|c|}{ POAG } \\
\hline & & $\begin{array}{l}\text { Peak } \\
\text { OPP }\end{array}$ & $\begin{array}{l}\text { Trough } \\
\text { OPP }\end{array}$ & $\begin{array}{l}\text { OPP } \\
\text { Fluctuation }\end{array}$ & $\begin{array}{l}\text { Mean } \\
\text { OPP }\end{array}$ & $\begin{array}{l}\text { Peak } \\
\text { OPP }\end{array}$ & $\begin{array}{l}\text { Trough } \\
\text { OPP }\end{array}$ & $\begin{array}{l}\text { OPP } \\
\text { Fluctuation }\end{array}$ & $\begin{array}{l}\text { Mean } \\
\text { OPP }\end{array}$ & $\begin{array}{l}\text { Peak } \\
\text { OPP }\end{array}$ & $\begin{array}{l}\text { Trough } \\
\text { OPP }\end{array}$ & $\begin{array}{l}\text { OPP } \\
\text { Fluctuation }\end{array}$ \\
\hline$\leq 1 \mathrm{mmHg}$ & 24.1 & 31.5 & 31.5 & 16.7 & 38.8 & 31.3 & 25.4 & 14.9 & 31.5 & 24.1 & 24.1 & 13.0 \\
\hline$\leq 2 \mathrm{mmHg}$ & 66.7 & 63.0 & 63.0 & 42.6 & 70.1 & 52.2 & 53.7 & 32.8 & 55.6 & 48.1 & 53.7 & 33.3 \\
\hline$\leq 3 \mathrm{mmHg}$ & 88.9 & 81.5 & 79.6 & 63.0 & 85.1 & 65.7 & 74.6 & 46.3 & 71.4 & 63.0 & 72.2 & 55.6 \\
\hline$\leq 4 \mathrm{mmHg}$ & 94.4 & 85.2 & 87.0 & 88.9 & 92.5 & 77.6 & 85.1 & 61.2 & 87.0 & 74.1 & 85.2 & 64.8 \\
\hline$\leq 5 \mathrm{mmHg}$ & 98.1 & 88.9 & 92.6 & 92.6 & 95.5 & 82.1 & 94.0 & 71.6 & 100 & 75.9 & 92.6 & 75.9 \\
\hline$\leq 6 \mathrm{mmHg}$ & 100 & 94.4 & 98.1 & 96.3 & 95.5 & 89.6 & 97.0 & 82.1 & & 83.3 & 96.3 & 87.0 \\
\hline$\leq 7 \mathrm{mmHg}$ & & 94.4 & 100 & 96.3 & 97.0 & 91.0 & 98.5 & 85.1 & & 92.6 & 100 & 90.7 \\
\hline$\leq 8 \mathrm{mmHg}$ & & 96.3 & & 98.1 & 100 & 92.5 & 100 & 85.1 & & 100 & & 92.6 \\
\hline
\end{tabular}

Data are expressed as percentage

OPP ocular perfusuion pressure, NTG normal tension glaucoma, POAG primary open-angle glaucoma

the same time points. The treated POAG patients did not manifest a repeatable diurnal IOP pattern across 2 visits spaced 1 week apart [15]. Hatanaka et al. measured the IOPs of ocular hypertension (OHT) and POAG patients with a GAT at 8:00, 11:00, 14:00 and 16:00 over 2 consecutive days. The diurnal mean IOP, peak IOP and trough IOP exhibited excellent reproducibility, but the IOP fluctuation exhibited fair reproducibility [16]. Song et al. measured the IOPs of healthy volunteers with a GAT and a Tono-Pen AVIA tonometer every $3 \mathrm{~h}$ once per week for 5 consecutive weeks. The maximum IOP and minimum IOP as measured with the GAT exhibited excellent reproducibility, but the IOP fluctuation exhibited poor reproducibility [17]. Aptel et al. measured the IOPs of POAG patients with a GAT at 9:00, 10:00, $11: 00,12: 00,14: 00,15: 00,16: 00$ and 17:00 over 4 visits in 6 months. The POAG patients did not exhibit a reproducible diurnal IOP pattern from month to month [18]. Xu et al. measured the IOPs of POAG and OHT patients with a noncontact tonometer at 2-h intervals over 2 consecutive days. The IOPs at the different time points generally exhibited fair or poor reproducibility, and the 24-h IOP curve of a single day was not highly reproducible in the short-term. The POAG group exhibited excellent reproducibility in terms of the mean IOP but fair reproducibility in terms of the peak IOP and poor reproducibility in terms of the IOP fluctuation. The OHT group exhibited excellent reproducibilities of the mean IOP and peak IOP but fair reproducibility of the IOP fluctuation [19]. Our data are partly consistent with those of previous studies, and the differences might be attributable to different sample sizes, study populations, IOP measurement time points, body positions, measurement intervals and types of tonometer. Furthermore, the CCT fluctuates with time and exhibits a circadian fluctuation [24]. However, the 24$\mathrm{h}$ IOP measurements were not influenced by the CCT fluctuations in either the both treated or untreated glaucoma patients [25]. We did not measure the CCTs at each time point; thus, we were unable to evaluate whether the CCT fluctuations influenced the reproducibilities of the 24-h IOPs or OPPs.

The mean IOP change between the short- and longterm periods and the short-term peak IOP were associated with progression of glaucoma [26]. Small mean changes (i.e., $1 \mathrm{mmHg}$ for the mean, $2 \mathrm{mmHg}$ for the peak, and $0.5 \mathrm{mmHg}$ for the fluctuations) elicited major changes in the single measurements [26]. In this study, in approximately $60 \%$ the glaucoma patients, the test-

Table 7 Peak Intraocular Pressure and Ocular Perfusion Pressure $(\mathrm{mmHg})$

\begin{tabular}{|c|c|c|c|c|c|c|c|}
\hline & \multirow{2}{*}{$\begin{array}{l}\text { Time } \\
\text { point }\end{array}$} & \multicolumn{3}{|c|}{ Patients with peak IOP at time point (\%) } & \multicolumn{3}{|c|}{ Patients with peak OPP at time point (\%) ${ }^{a}$} \\
\hline & & Control & NTG & POAG & $\overline{\text { Control }}$ & NTG & POAG \\
\hline \multirow[t]{4}{*}{ Daytime } & 0900 & 14.8 & 16.4 & 20.4 & 11.1 & 10.4 & 5.6 \\
\hline & 1200 & 25.9 & 31.3 & 29.6 & 13.0 & 1.5 & 7.4 \\
\hline & 1500 & 11.1 & 9.0 & 9.3 & 9.3 & 6.0 & 14.8 \\
\hline & 1800 & 20.4 & 34.3 & 24.1 & 20.4 & 28.4 & 9.3 \\
\hline \multirow[t]{3}{*}{ Nighttime } & 2100 & 3.7 & 9.0 & 1.9 & 18.5 & 13.4 & 14.8 \\
\hline & 2400 & 3.7 & 9.0 & 9.3 & 13.0 & 28.4 & 27.8 \\
\hline & 0600 & 46.3 & 31.3 & 25.9 & 14.8 & 11.9 & 20.4 \\
\hline
\end{tabular}

Data are expressed as percentage

$I O P$ intraocular pressure, OPP ocular perfusion pressure, NTG normal tension glaucoma, POAG primary open-angle glaucoma

${ }^{a}$ The same peak pressure may have occurred at more than one time point 
Table 8 Maximum Daytime Pressures Measured within Different Intervals of Maximum Nighttime Pressures

\begin{tabular}{|c|c|c|c|c|c|c|}
\hline \multirow[t]{2}{*}{$\begin{array}{l}\text { Nighttime elevation above } \\
\text { daytime peak pressure }(\mathrm{mmHg})\end{array}$} & \multicolumn{3}{|c|}{$\begin{array}{l}\text { Per cent of daytime IOP }<\text { than indicated } \\
\text { level in column } 1(\%)\end{array}$} & \multicolumn{3}{|c|}{$\begin{array}{l}\text { Per cent of daytime OPP }<\text { than indicated } \\
\text { level in column } 1(\%)\end{array}$} \\
\hline & Control & NTG & POAG & Control & NTG & POAG \\
\hline$\leq 0$ & 51.9 & 44.8 & 38.9 & 53.7 & 47.8 & 40.7 \\
\hline+1 & 24.1 & 26.9 & 20.4 & 9.3 & 1.5 & 14.8 \\
\hline+2 & 9.3 & 16.4 & 33.3 & 14.8 & 13.4 & 11.1 \\
\hline+3 & 11.1 & 7.5 & 3.7 & 13 & 14.9 & 13 \\
\hline+4 & 1.9 & 4.8 & 3.7 & 5.6 & 11.9 & 7.4 \\
\hline$\geq 5$ & 1.9 & 0 & 0 & 3.7 & 10.4 & 13.0 \\
\hline
\end{tabular}

Data are expressed as percentage

IOP intraocular pressure, OPP ocular perfusion pressure, NTG normal tension glaucoma, POAG primary open-angle glaucoma

retest differences in the IOPs fell within $\pm 1 \mathrm{mmHg}$. Further studies are needed to assess the influence of IOP reproducibility on the progression of glaucoma.

Song et al. also calculated the diurnal OPPs of young volunteers every $3 \mathrm{~h}$ once per week for 5 consecutive weeks. The maximum OPP, minimum OPP and OPP fluctuation values exhibited excellent, fair and poor reproducibilities, respectively [17]. In this study, the peak OPP, trough OPP and OPP values at the time points exhibited good reproducibilities in the normal volunteers, but the OPP fluctuations exhibited poor reproducibility. Our data are partially consistent with the findings of Song et al. The differences might be due to the different sample sizes (54 subjects vs. 10 subjects), study populations (Chinese vs. young female Koreans) and measurement intervals ( 2 consecutive days vs. 5 consecutive weeks).

In this study, the POAG group exhibited a significantly lower OPP than the NTG patients and normal volunteers. A reduction of OPP is indicative of a reduction in the vascular flow to the optic nerve and could lead to glaucomatous optic nerve damage $[13,27]$. Sehi et al. reported that a relative change in the diurnal mean OPP is a risk factor for the diagnosis of POAG [9]. Choi et al. reported that marked circadian mean OPP fluctuations might be a risk factor for the development of NTG [6]. Later, Choi et al. reported that a greater circadian mean OPP is significantly related to a decreased MD, an increased pattern SD, an increased Advanced Glaucoma Intervention Study (AGIS) score, a reduced temporal, superior, nasal, inferior, and temporal (TSNIT) average, a reduced inferior average, and an increased nerve fibre indicator on scanning laser polarimetry [7]. Sung et al. reported on 101 NTG patients who underwent 24 -h sitting IOP and OPP measurements over more than 4 years. The 24-h OPP fluctuations were found to be significant predictors of VF progression [8]. Therefore, OPP has been found to be a risk factor for the severity and progression of glaucoma in previous studies. In the present study, the IOP/OPP fluctuations in the glaucoma patients were not highly reproducible. Only the peak OPP of the POAG patients occurred at same time point (24:00) in the diurnal curves from the 2 consecutive days. Previous studies might not have comprehensively demonstrated the relevance of IOP/OPP to glaucoma. Moreover, different equations have been used to calculate the OPP; OPP has also been defined as ([DBP-1/3 (SBP-DBP)]-IOP) [21]. Different methods of calculation would lead to different results. Twenty-four-hour OPP fluctuations may be associated with nocturnal BP reductions $[8,28]$. We did not analyse the relationship between the reproducibility of OPP fluctuations and nocturnal BP reductions. Further studies are needed to identify the factors that influence the reproducibility of OPP measurements.

There are some limitations in this study. We did not measure the IOP at 3:00 over the 2 consecutive days. This measurement would have required us to wake up the subjects, which would have disturbed their sleep-wake rhythm. This strategy might have affected the IOP and BP measurements at the later time points. Recently, a wireless contact lens sensor (CLS) was used to measure IOPs over $24 \mathrm{~h}$, and this approach did not require the waking of sleeping subjects [29]. Applanation resonance tonometers are less affected by corneal properties than GATs and exhibit good inter-examiner reproducibility and intra-examiner repeatability [30]. Shortterm IOP measurements and long-term IOP measurements will be more comfortably and easily realized in the future. All of the IOP and BP measurements were not performed by a single doctor, but the IOP and BP measurements within each day were performed by single experienced doctors.

\section{Conclusion}

The IOP measurements within a single day were not highly reproducible in the short-term. This report is the first to document the reproducibility of OPP measurements in a different study population. The OPP measurements exhibited better reproducibilities than the IOP measurements. The normal volunteers exhibited better IOP and OPP reproducibilities than the glaucoma patients. The IOP and OPP fluctuations exhibited fair or poor reproducibilities; therefore, IOP and OPP fluctuations cannot be assessed based on IOP and OPP measurements collected within a single day. 


\section{Acknowledgments}

None.

\section{Funding}

This study was supported by Natural Science Foundation of Tianjin Municipal Science \& Technology Commission (Grant No. 13JCYBJC39900).

\section{Availability of data and materials}

All the data supporting our findings is contained within the manuscript.

\section{Authors' contributions}

Y Gao has been involved in designing the study and acquisition of the most data, and drafting the manuscript of this study. B Wan and P Li made contributions in analyzing the data and revising the manuscript. $Y$ Zhang made contributions in IOP and BP measurements. X Tang supervised the study designs, analyzed and interpreted the results, and revised manuscript draft. All authors read and approved the final manuscript.

\section{Competing interests}

The authors declare that they have no competing interests.

\section{Consent for publication}

Not applicable.

\section{Ethics and consent to participate}

The study was approved by the institutional review board and Ethics Committee of Tianjin Eye Hospital (IRB approval NO. TJYYLL-2014-15). Written informed consent was obtained from all participants.

\section{Financial support}

Natural Science Foundation of Tianjin Municipal Science \& Technology Commission (Grant No. 13JCYBJC39900)

\section{Author details}

${ }^{1}$ Tianjin Eye Hospital, Tianjin Key Laboratory of Ophthalmology and Vision Science, Clinical College of Ophthalmology, Tianjin Medical University, 4, Gansu Road, Heping District, Tianjin 300020, People's Republic of China. 2Department of Laboratory, Central Hospital Affiliated to Shenyang Medical College, Shenyang, China. ${ }^{3}$ Department of Ophthalmology, the Second Hospital Affiliated to Dalian Medical University, Dalian, China.

Received: 24 January 2016 Accepted: 16 June 2016

\section{Published online: 18 August 2016}

\section{References}

1. The Advanced Glaucoma Intervention Study (AGIS): 7. The relationship between control of intraocular pressureand visual field deterioration.The AGIS Investigators. Am J Ophthalmol. 2000 130: 429-40.

2. Konstas AG, Irkec MT, Teus MA, Cvenkel B, Astakhov YS, Sharpe ED, et al. Mean intraocular pressure and progression based on corneal thickness in patients with ocular hypertension. Eye. 2009;23:73-8.

3. Nouri-Mahdavi K, Hoffman D, Coleman AL, Liu G, Li G, Gaasterland D, et al. Predictive factors for glaucomatous visual field progression in the advanced glaucoma intervention study. Ophthalmology. 2004;111:1627-35.

4. Stewart WC, Kolker AE, Sharpe ED, Day DG, Konstas AG, Hollo G, et al. Longterm progression at individual mean intraocular pressure levels in primary open-angle and exfoliative glaucoma. Eur J Ophthalmol. 2008;18:765-70.

5. Meyer JH, Brandi-Dohrn J, Funk J. Twenty four hour blood pressure monitoring in normal tension glaucoma. Br J Ophthalmol. 1996;80:864-7.

6. Choi J, Jeong J, Cho HS, Kook MS. Effect of nocturnal blood pressure reduction on circadian fluctuation of mean ocular perfusion pressure: a risk factor for normal tension glaucoma. Invest Ophthalmol Vis Sci. 2006;47:831-6.

7. Choi J, Kim KH, Jeong J, Cho HS, Lee CH, Kook MS. Circadian fluctuation of mean ocular perfusion pressure is a consistent risk factor for normal-tension glaucoma. Invest Ophthalmol Vis Sci. 2007:48:104-11.

8. Sung KR, Lee S, Park SB, Choi J, Kim ST, Yun SC, et al. Twenty-four hour ocular perfusion pressure fluctuation and risk of normal-tension glaucoma progression. Invest Ophthalmol Vis Sci. 2009;50:5266-74.
9. Sehi M, Flanagan JG, Zeng L, Cook RJ, Trope GE. Relative change in diurnal mean ocular perfusion pressure: a risk factor for the diagnosis of primary open-angle glaucoma. Invest Ophthalmol Vis Sci. 2005;46:561-7.

10. Liu JH, Zhang X, Kripke DF, Weinreb RN. Twenty-four-hour intraocular pressure pattern associated with early glaucomatous changes. Invest Ophthalmol Vis Sci. 2003;44:1586-90.

11. Hermida RC, Ayala DE, Portaluppi F. Circadian variation of blood pressure: the basis for the chronotherapy of hypertension. Adv Drug Deliv Rev. 2007:59:904-22.

12. Tielsch JM, Katz J, Sommer A, Quigley HA, Javitt JC. Hypertension, perfusion pressure, and primary open-angle glaucoma: a population-based assessment. Arch Ophthalmol. 1995;113:216-21.

13. Bonomi L, Marchini G, Marraffa M, Bernardi P, Morbio R, Varotto A. Vascular risk factors for primary open angle glaucoma: the egna-neumarkt study. Ophthalmology. 2000:107:1287-93.

14. Realini T, Weinreb RN, Wisniewski SR. Diurnal intraocular pressure patterns are not repeatable in the short term in healthy individuals. Ophthalmology. 2010;117:1700-4.

15. Realini T, Weinreb RN, Wisniewski S. Short-term repeatability of diurnal intraocular pressure patterns in glaucomatous individuals. Ophthalmology. 2011;118:47-51.

16. Hatanaka M, Babic M, Susanna Jr R. Reproducibility of the mean fluctuation, and IOP peak in the diurnal tension curve. J Glaucoma. 2013;22:390-2.

17. Song YK, Lee CK, Kim J, Hong S, Kim CY, Seong GJ. Instability of 24-hour intraocular pressure fluctuation in healthy young subjects: a prospective, cross-sectional study. BMC Ophthalmol. 2014;14:127.

18. Aptel F, Lesoin A, Chiquet C, Aryal-Charles N, Noel C, Romanet JP. Longterm reproducibility of diurnal intraocular pressure patterns in patients with glaucoma. Ophthalmology. 2014;121:1998-2003.

19. Xu S, Jiao Q, Cheng Y, Sun J, Lu Q, Zhong Y. Short-term reproducibility of twenty-four-hour intraocular pressure curves in untreated patients with primary open-angle glaucoma and ocular hypertension. PLoS One. 2015; 10(10):e0140206.

20. Quaranta L, Konstas AG, Rossetti L, Garcia-Feijoo J, O'Brien C, Nasr MB, et al. Untreated 24-h intraocular pressures measured with goldmann applanation tonometry vs nighttime supine pressures with Perkins applanation tonometry. Eye. 2010;24:1252-8.

21. Quaranta L, Katsanos A, Russo A, Riva I. 24-hour intraocular pressure and ocular perfusion pressure in glaucoma. Surv Ophthalmol. 2013;58:26-41.

22. Landis JR, Koch GG. The measurement of observer agreement for categorical data. Biometrics. 1977;33:159-74.

23. Colombo L, Fogagnolo P, Montesano G, De Cillà S, Orzalesi N, Rossetti L. Strategies to estimate the characteristics of 24-hour IOP curves of treated glaucoma patients during office hours. BMC Ophthalmol. 2016;16:15.

24. Fogagnolo P, Rossetti L, Mazzolani F, Orzalesi N. Circadian variations in central corneal thickness and intraocular pressure in patients with glaucoma. Br J Ophthalmol. 2006;90:24-8.

25. Fogagnolo P, Capizzi F, Orzalesi N, Figus M, Ferreras A, Rossetti L. Can mean central corneal thickness and its 24-hour fluctuation influence fluctuation of intraocular pressure? J Glaucoma. 2010;19:418-23.

26. Fogagnolo P, Orzalesi N, Centofanti M, Oddone F, Manni G, Rossetti L. Short- and long-term phasing of intraocular pressure in stable and progressive glaucoma. Ophthalmologica. 2013;230:87-92.

27. Liu JH, Kripke DF, Twa MD, Hoffman RE, Mansberger SL, Rex KM, et al. Twenty-four-hour pattern of intraocular pressure in the aging population. Invest Ophthalmol Vis Sci. 1999:40:2912-7.

28. Rossetti L, Sacchi M, Karabatsas CH, Topouzis F, Vetrugno M, Centofanti M, et al. Comparison of the effects of bimatoprost and a fixed combination of latanoprost and timolol on 24-hour blood and ocular perfusion pressures: the results of a randomized trial. BMC Ophthalmol. 2015;15:7.

29. Mansouri K, Weinreb RN, Liu JH. Efficacy of a contact lens sensor for monitoring 24-H intraocular pressure related patterns. PLoS One. 2015; 10(5):e0125530.

30. Ottobelli L, Fogagnolo P, Frezzotti P, De Cillà S, Vallenzasca E, Digiuni M, et al. Repeatability and reproducibility of applanation resonance tonometry: a cross-sectional study. BMC Ophthalmol. 2015;15:36. 